\title{
The Practical Aspects of ToF-SIMS Analysis in the Industrial and Contract Laboratory Setting
}

\author{
Birgit Hagenhoff, Elke Tallarek, Michael Fartmann, and Reinhard Kersting
}

Tascon GmbH, Mendelstrasse 17, 48149 Muenster, Germany

Time-of-Flight Secondary Ion Mass Spectrometry (ToF-SIMS) has emerged as an important analytical tool supporting product development and problem solving for customers in the automotive, coatings, electronics, glass, life sciences, and pharmaceutical markets. Typical analyses include the chemical characterization of surfaces, lateral imaging, depth profiling, identification of trace impurities, quality control, and patent litigation. In general, any successful analytical measurement requires highly reproducible and accurate data. The development and the implementation of standard operating protocols (SOP) or, the more rigorous ISO 17025 accreditation, helps ensure data quality and integrity [1]. The objective of this paper is to highlight the best practices that analytical service providers can use to ensure data quality. These best practices can include monitoring instrument performance, automated procedures for data acquisition, appropriate use of standards and reference materials, and the development and use of spectra libraries for data interpretation.

\section{Monitoring Instrument Performance}

Producing high quality ToF-SIMS data starts with ensuring the instrument is ready for analysis, that personnel are well trained, and that analysis procedures have the flexibility to adapt to real world samples. Commercial aluminum foil and polycarbonate serve as suitable, low-cost references to monitor instrument performance. Here, data are typically collected at least weekly (e.g., after system bakeout). For example, the relative intensity of common organic contamination (e.g., silicones, phthalates, and fluorocarbons) is monitored to ensure vacuum quality. Analysis of an insulating sample such as polycarbonate is also necessary to ensure analyzer sensitivity and appropriate charge compensation. Figure 1 shows a sample control chart for the normalized intensity of the $\mathrm{C}_{14} \mathrm{H}_{11} \mathrm{O}_{2}{ }^{-}$peak monitored over several weeks. A normalized intensity within $2 \sigma$ of the average is considered acceptable. The low normalized intensity starting around week 15 corresponds to a channel-plate failure and the high normalized intensity around week 118 corresponds to a deviation in the standard acquisition parameters.

\section{Development of Depth Profiling Standards and References}

An exciting advancement in recent years has been the development of cluster primary ion sources $\left(\mathrm{Au}_{\mathrm{n}}{ }^{+}\right.$, $\mathrm{Bi}_{\mathrm{n}}{ }^{+}, \mathrm{SF}_{5}{ }^{+}, \mathrm{C}_{60}{ }^{+}$, or $\left.\mathrm{Ar}_{\mathrm{n}}{ }^{+}\right)$[2,3] for ToF-SIMS analysis. $\mathrm{C}_{60}{ }^{+}$and $\mathrm{Ar}_{\mathrm{n}}{ }^{+}$primary sources have been particularly important in the development molecular depth profiling. In the same way a multilayer standard such as epitaxial SiGe (FZ Jülich) serves as reference for elemental depth profiling with $\mathrm{O}_{2}$ or $\mathrm{Cs}$, organic multilayer standards and references must be developed for molecular depth profiling. At the present time, the Irganox 1010/Irganox 3114 multilayer standard developed by the National Physical Laboratory serves as a reference for understanding sputter rates and depth resolution [4]. Figure 2 shows the $2.5 \mathrm{keV} \mathrm{Ar}{ }_{1700}$ GCIB depth profile of the NPL standard. A depth resolution of $5.0 \mathrm{~nm}$ FWHM is well maintained through the profile down to the fourth delta layer. 


\section{References:}

[1] J. D. Honsa and D. A. McIntyre, J. of AOCA International, 86 (2003), p. 1038.

[2] S. Rabbani, A. M. Barber, J. S. Fletcher, N. P. Lockyer, J. C. Vickerman, Anal. Chem., 83 (2011), p. 3793.

[3] C. M Mahoney, Mass Spectrometry Reviews, Wiley Periodicals, Inc. (2009).

[4] P. J. Cumpson, J. F. Portoles, N. Sano, J. Vac. Sci. A, 31 (2013).

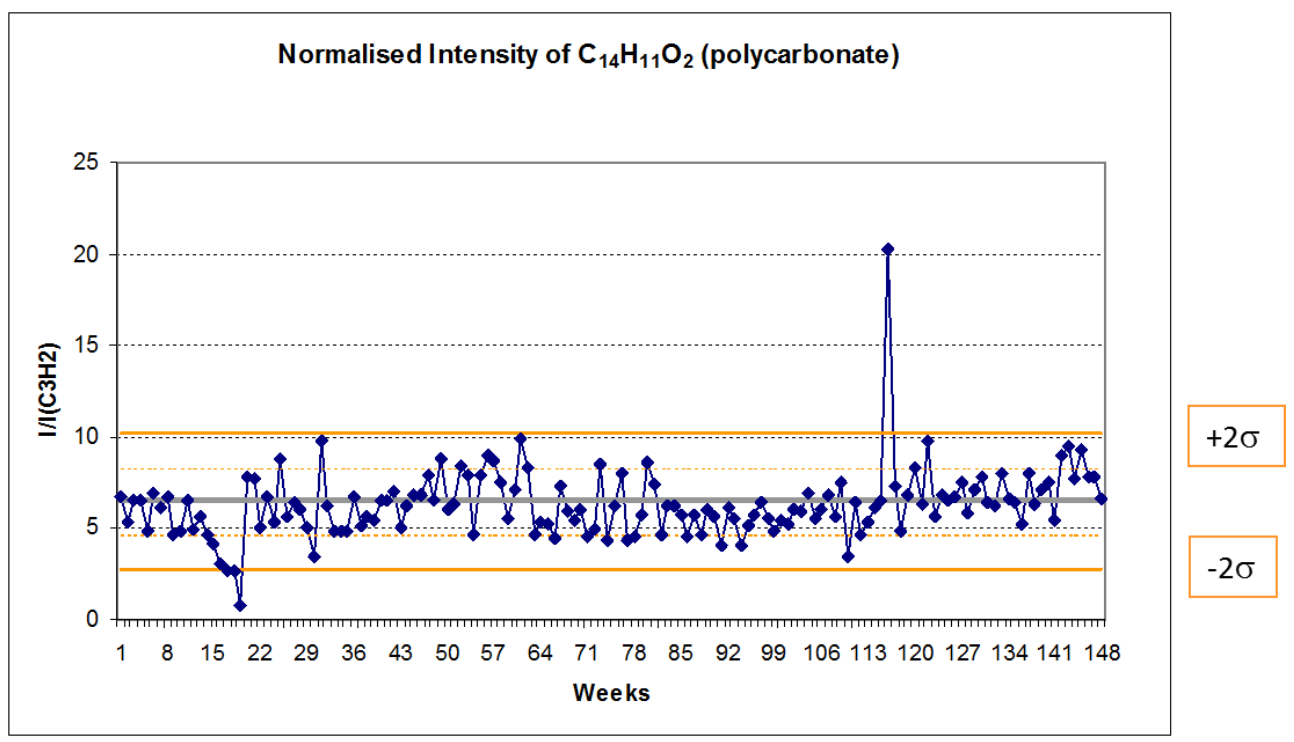

Figure 1. Control chart for polycarbonate reference.
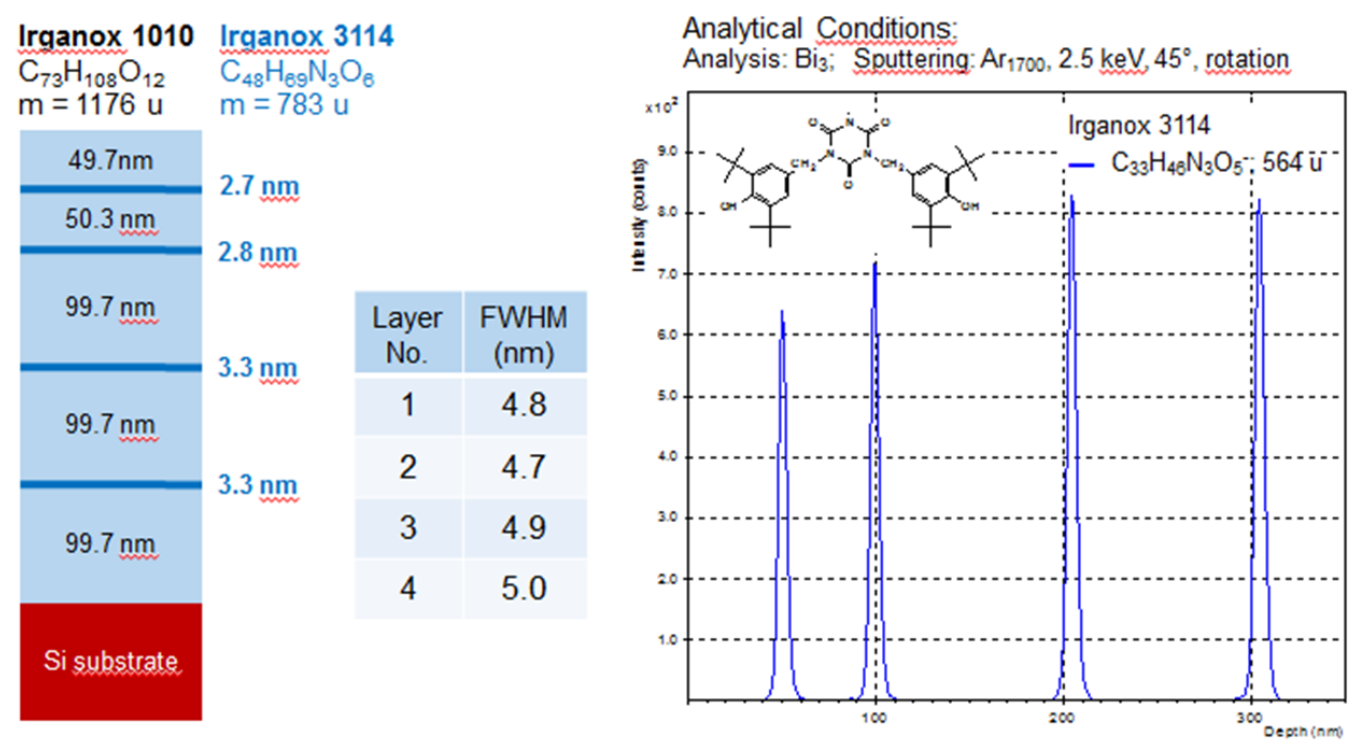

Figure 2. Depth profile of National Physical Laboratory organic multilayer standard: VAMAS study of organic depth profiling. Courtesy of ION-TOF. 